\title{
Molecular Rationale for Improved Dynamic Nuclear Polarization of Biomembranes
}

\author{
Adam N. Smith, ${ }^{1,2}$ Umar T. Twahir, ${ }^{1}$ Thierry Dubroca, ${ }^{2}$ Gail E. Fanucci, ${ }^{1}$ Joanna R. Long $*^{2,3}$ \\ ${ }^{1}$ Department of Chemistry, University of Florida, 214 Leigh Hall, P.O. Box 117200, Gainesville,
} FL 32611

\footnotetext{
${ }^{2}$ National High Magnetic Field Laboratory, 1800 E. Paul Dirac Dr., Tallahassee, FL 32310

${ }^{3}$ Department of Biochemistry \& Molecular Biology, University of Florida, P.O. Box 100245, Gainesville, FL, 32610
}

SUPPORTING INFORMATION 


\section{DNP Enhancement as a function of lipid deuteration}

Cross effect DNP relies on nuclear spin diffusion to transfer polarization throughout the NMR active nuclei in the sample of interest. Specifically, for ${ }^{13} \mathrm{C}$ CP-based experiments with biomolecular samples, polarization is distributed throughout the sample via ${ }^{1} \mathrm{H}^{-}{ }^{1} \mathrm{H}$ spin diffusion. However, high levels of protonation can lead to deleterious relaxation processes due to strong ${ }^{1} \mathrm{H}-{ }^{1} \mathrm{H}$ dipolar couplings. Thus, it is important to strike a balance in protonation levels that leads to efficient ${ }^{1} \mathrm{H}-{ }^{1} \mathrm{H}$ spin diffusion while minimizing non-productive relaxation effects. It was previously determined, for aqueous samples, that a solvent system of $90: 10 \mathrm{D}_{2} \mathrm{O}: \mathrm{H}_{2} \mathrm{O}$ leads to the most efficient DNP transfer. In liposome based samples, the acyl chains of the lipids result in an

additional ${ }^{1} \mathrm{H}$ spin bath, so we therefore varied the protonation levels of the lipids by utilizing different amounts of DPPC with deuterated acyl chains $\left(\right.$ DPPC- $\left.\mathrm{d}_{62}\right)$, where all C-H bonds are replaced with C-D bonds. Liposomes composed of 1:9, 1:1, or 9:1 DPPC- $\mathrm{d}_{62}:$ POPC containing 3 mol\% TEMPO-PC as the polarization agent were made and DNP enhancement of the ${ }^{13} \mathrm{C}$ resonance resulting from the lipid acyl chain was measured. The 1:9 and 9:1 DPPC- $\mathrm{d}_{62}:$ POPC samples resulted in DNP enhancements of 4.5 and 4.0 respectively (Figure-S1). In contrast, the 1:1 DPPC- $\mathrm{d}_{62}:$ POPC sample resulted in a DNP enhancement of 7.0. Based on these results all other liposome samples were made with 1:1 DPPC- $\mathrm{d}_{62}:$ POPC. 


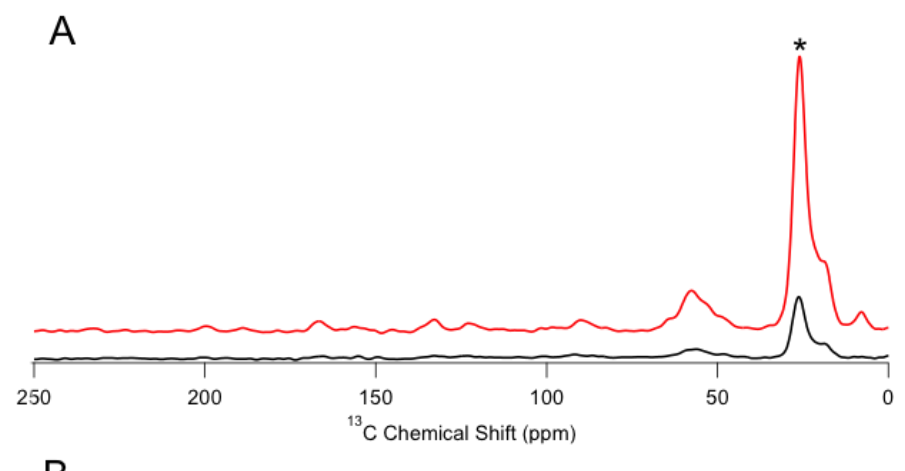

B
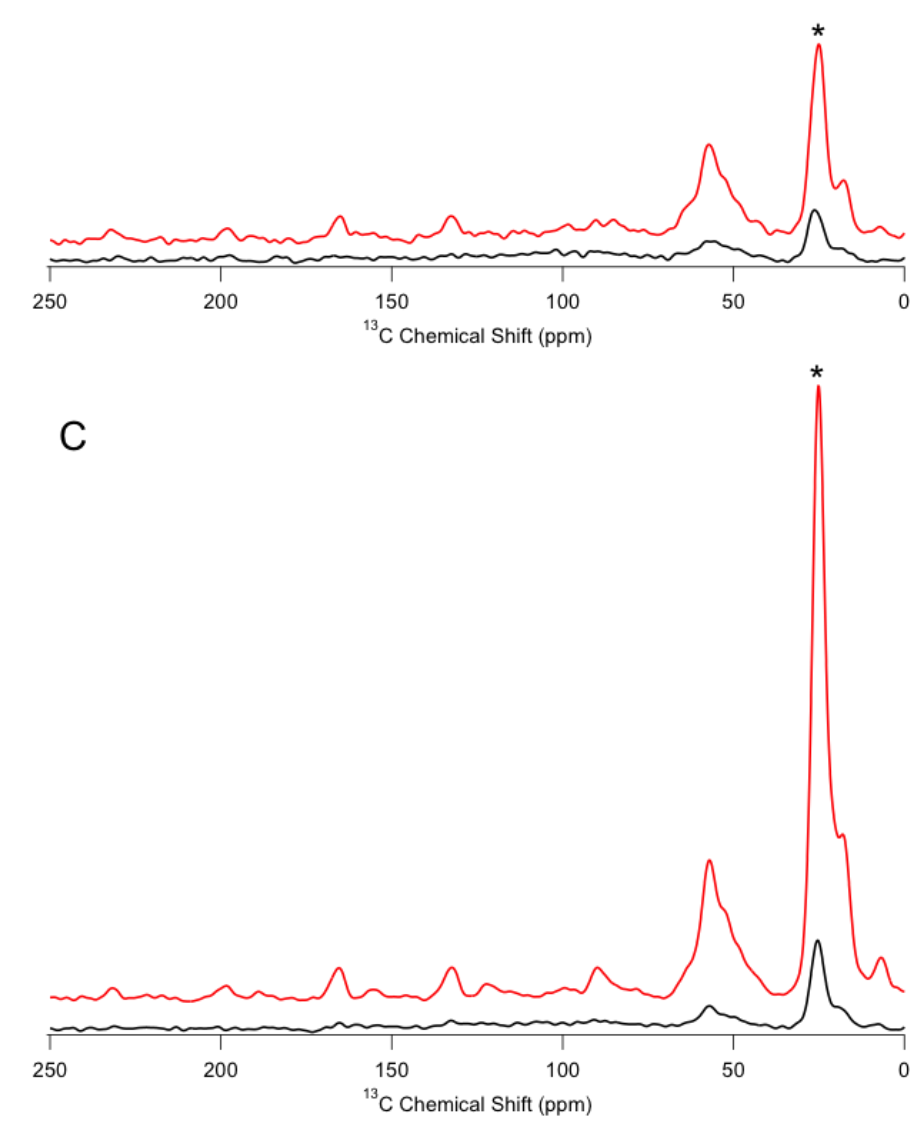

Figure S1. ${ }^{13} \mathrm{C}$ CP MAS spectra with the MW on (red) and off (black) of liposomes composed of A) 1:9 DPPC-d $\mathrm{d}_{62}:$ POPC, B) 9:1 DPPC-d $\mathrm{d}_{62}:$ POPC, and C) 1:1 DPPC- $\mathrm{d}_{62}:$ POPC each with $3 \mathrm{~mol} \%$ TEMPO-PC as the polarization agent. DNP enhancement was measured from the acyl chain resonance, indicated with * in the spectra. 


\section{DNP Enhanced ${ }^{13} \mathrm{C}$ CP MAS ssNMR Spectra of Liposomes with Varying SL-lipids}

The DNP enhancements reported in Figures $3 \mathrm{~A}$ and $4 \mathrm{~A}$ were measured using the DNP enhanced ${ }^{13} \mathrm{C}$ CP MAS ssNMR spectra given in Figures S2 and S3.
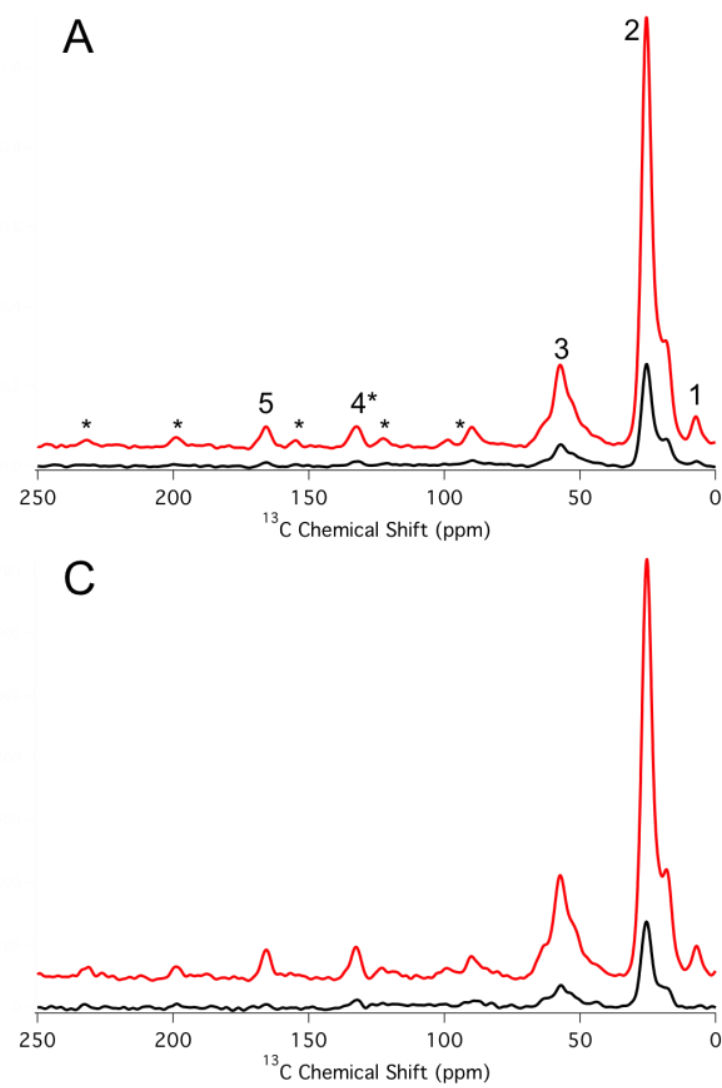

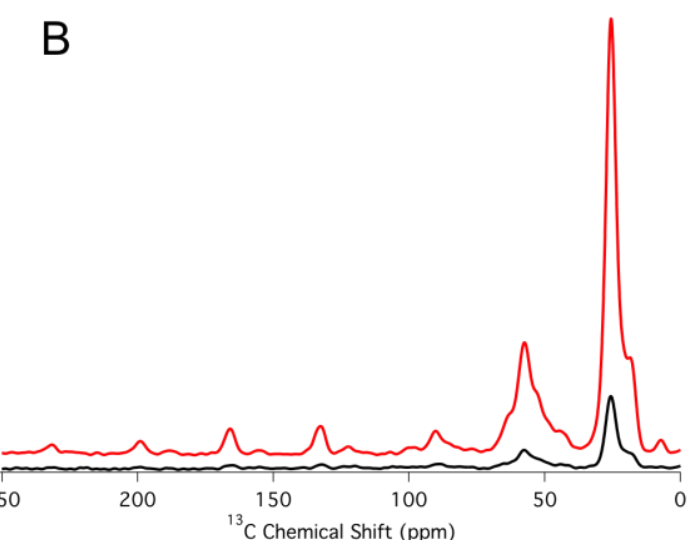

D

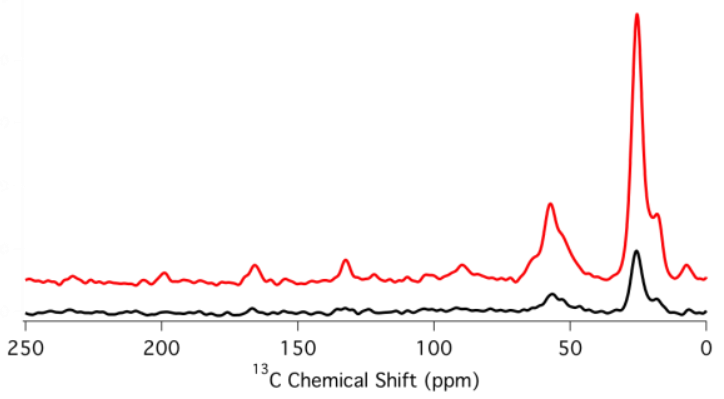

Figure S2: ${ }^{13} \mathrm{C}$ CP MAS ssNMR spectra of liposomes with the MW on (red) and off (black) were recorded as described in DNP MAS ssNMR section of the Materials \& Methods. The polarization agent 7-Doxyl-PC was used at 1.5 (A), 3.0 (B), 6.0 (C), and 8.0 (D) mol\% of total lipid. The resonances were assigned as 1 terminal methyl of lipid acyl chain, $2 \mathrm{sp}^{3}$ carbons of the lipid acyl chain, 3 lipid glycerol backbone, $4^{*} \mathrm{sp}^{2}$ carbons at the point of unsaturation in the lipid acyl chain which also includes the spinning sideband from 5,5 lipid carbonyl groups, and * spinning sidebands resulting from 3 and 5 . The lipid acyl chain (resonance 2) was used to measure all reported DNP enhancements. 

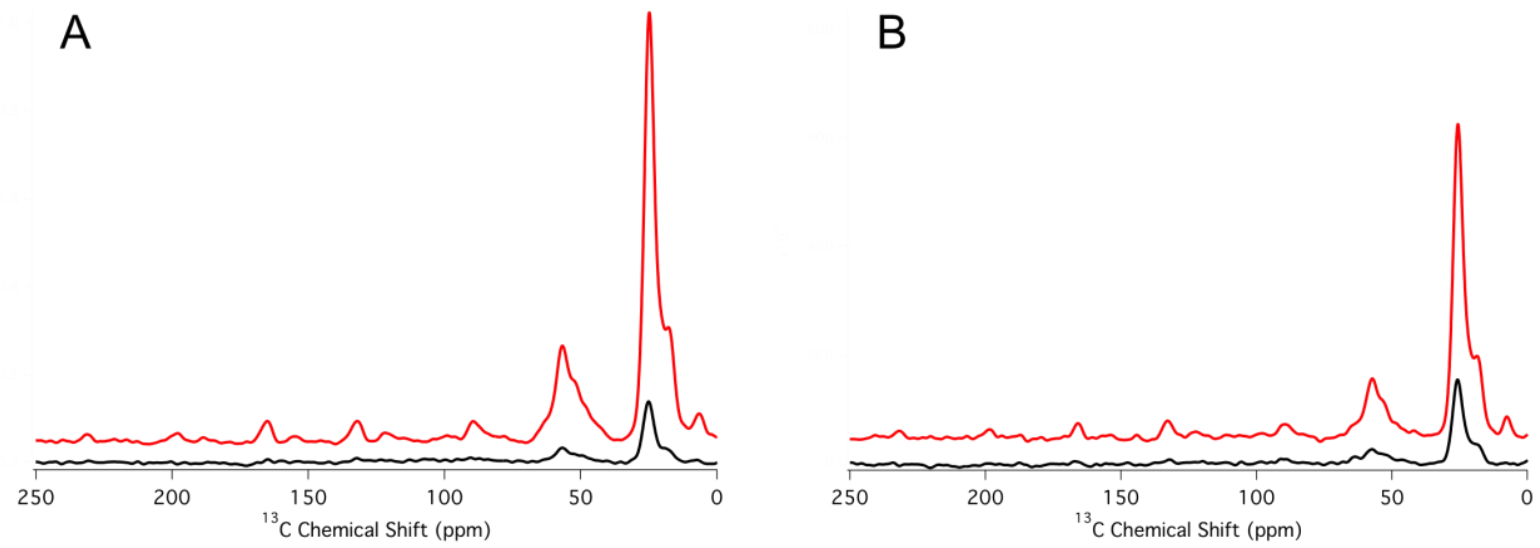

Figure S3: ${ }^{13} \mathrm{C}$ CP MAS ssNMR spectra of liposomes with the MW on (red) and off (black) were recorded as described in DNP MAS ssNMR section of the Materials \& Methods. The polarization agent TEMPO-PC (A), 16-Doxyl-PC (B) were used at $3 \mathrm{~mol} \%$ of total lipid. The same resonance assignments as in Fig. S2A were used and the lipid acyl chain (resonance 2) was used to measure all reported DNP enhancements.

\section{DNP Buildup Time}

DNP buildup times for the observed liposome resonances with the use of TOTAPOL were measured and are reported in Figure S4 and Table S1. We note that the DNP buildup times should be slightly longer when TOTAPOL is used as the polarization agent compared to SLlipids due to the distance dependence of polarization propagation via nuclear spin diffusion. The $600 \mathrm{MHz} / 395 \mathrm{GHz}$ DNP system at the National High Magnetic Field Laboratory utilizes a unique quasi-optical system for MW transmission (manuscript in preparation). This quasi-optical system allows for insertion of various active and passive components in-line with the MW beam. Specific to this work, a mechanical shutter that can open and close on a millisecond timescale was put in-line with the MW beam, the shutter is triggered by the NMR spectrometer where open/close commands are built into the NMR pulse program and DNP buildup times can be directly measured by gating the MW on for a given amount of time. 


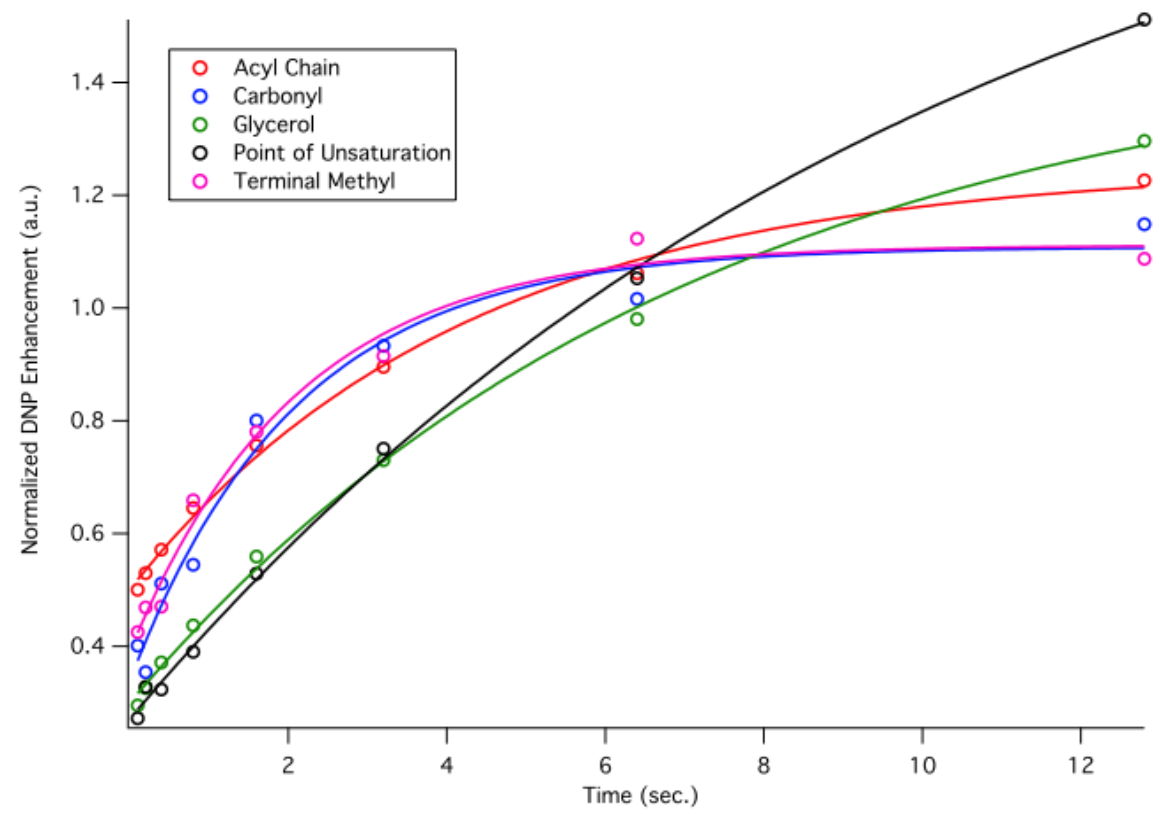

Figure S4: DNP buildup curves are shown for liposomes and TOTAPOL polarization agent. Note that the glycerol curve includes resonances resulting from solvent glycerol and the glycerol backbone of phospholipids.

Table S1: DNP buildup times for liposome resonances

\begin{tabular}{ll}
\hline Resonance & DNP Buildup Time (sec.) \\
\hline glycerol (solvent + lipid backbone) & 7.2 \\
carbonyl & 2.1 \\
acyl chain & 4.2 \\
point of unsaturation & 11 \\
terminal methyl & 2.1 \\
\hline
\end{tabular}




\section{FWHH Measurements}

Table S2: FWHH measurements

\begin{tabular}{|c|c|c|c|c|}
\hline SL-lipid & Resonance & \multicolumn{3}{|c|}{ FWHH (Hz) } \\
\hline & & 7-Doxyl-PC & TEMPO-PC & 16-Doxyl-PC \\
\hline \multirow{3}{*}{$1.5 \mathrm{~mol} \%$} & acyl chain & 670 & & \\
\hline & glycerol backbone & 1187 & & \\
\hline & carbonyl & 646 & & \\
\hline \multirow[t]{3}{*}{$3.0 \mathrm{~mol} \%$} & acyl chain & 696 & 693 & 685 \\
\hline & glycerol backbone & 1311 & 1373 & 1234 \\
\hline & carbonyl & 675 & 676 & 537 \\
\hline \multirow[t]{3}{*}{$6.0 \mathrm{~mol} \%$} & acyl chain & 699 & & \\
\hline & glycerol backbone & 1231 & & \\
\hline & carbonyl & 601 & & \\
\hline \multirow[t]{3}{*}{$8.0 \mathrm{~mol} \%$} & acyl chain & 708 & & \\
\hline & glycerol backbone & 1323 & & \\
\hline & carbonyl & 636 & & \\
\hline
\end{tabular}

The supporting information are available free of charge. 